Supporting Information

\title{
Selective Molecular Sieving through a Large Graphene Nanopore with Surface Charges
}

Chengzhen Sun*, Shaohua Zhu, Maochang Liu, Shaohua Shen, Bofeng Bai

State Key Laboratory of Multiphase Flow in Power Engineering, Xi'an Jiaotong University, Xi'an, Shaanxi 710049, China

*Email: sun-cz@,xjtu.edu.cn

\section{Content}

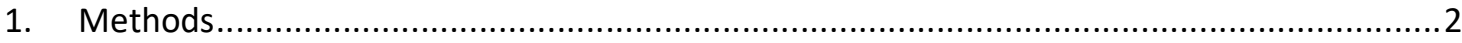

1.1 Graphene nanopores and surface charges ....................................................

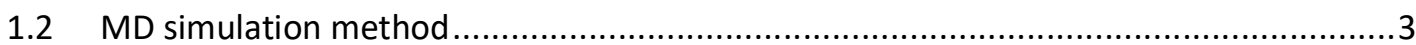

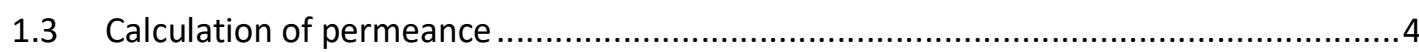

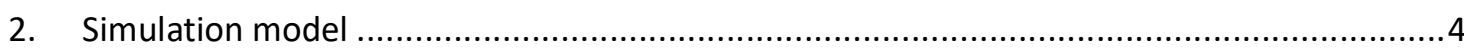

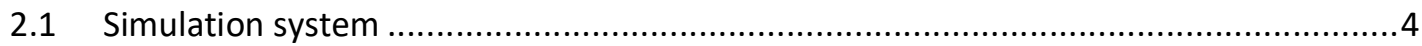

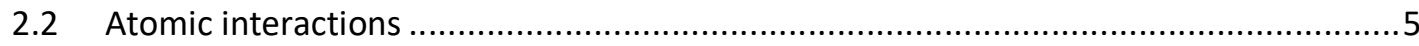

2.3 Harmonic potential.............................................................................

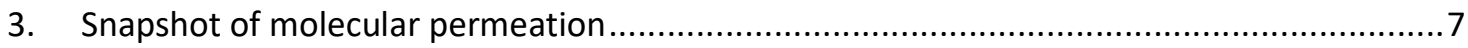

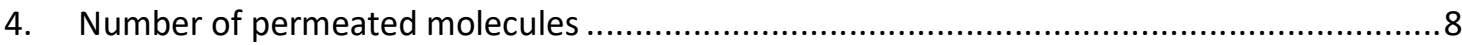

5. Molecular number density distribution on the graphene surface ................................10

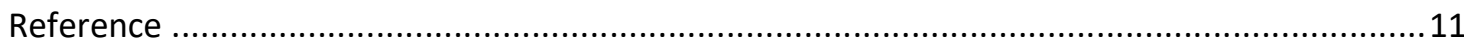




\section{Methods}

\subsection{Graphene nanopores and surface charges}

In this study, an all-H passivated nanopore is adopted to reveal the effects of surface charge density on the selectivity of $\mathrm{CO}_{2}$ molecules over $\mathrm{N}_{2}$ molecules. The nanopore is created based on a 16-graphene-ring-units pore with a near-circular shape. After the all-H passivation, the pore diameter is estimated as $0.52 \mathrm{~nm}$ by averaging the longest and the shortest distance between a pair of pore-rim $\mathrm{H}$ atoms of which the connecting line crosses the pore center. The pore configuration is shown in Figure 1(b). Only one nanopore appears in the graphene sheet with an area of $4 \times 4 \mathrm{~nm}^{2}$, such that the pore density in the graphene sheet is $6.25 \times 10^{12} \mathrm{~cm}^{2}$. The pore density in this simulation is comparable to those in the experimentally fabricated NPG membranes ${ }^{1-4}$.

In practice, the graphene nanopores with surface charges can be tried to be realized by either connecting with a negative electrode or introducing of anionic groups on the surfaces, such that the charge distribution and migration are very complicated. In order to simply model the charge-loaded graphene nanopores in the classical MD simulations, a negative charge of the same value is added to each carbon atom of graphene. The uniformly distributed charges are adopted by considering that the surface charges can be added by evenly introducing the anionic groups on the graphene surfaces. Meanwhile, during the simulations the charges are assumed to be static and the charge migration is totally neglected. The surface charge density is just varied by adjusting the value of partial charges on the graphene carbon atoms. The partial charge varies from 0.0 e to $0.15 \mathrm{e}$; accordingly, the range of surface charge density is 0.00 to $-5.934 \mathrm{e} / \mathrm{nm}^{2}$; such charge density is considered to be practicable by introducing the anionic groups with a high valence. The simplifications in the modelling of graphene nanopores with surface charges may inevitably cause some discrepancies comparing to the real systems, but we consider that the selectivity improvement via surface charges can be well interpreted in the simulations owing to the dominating role of the electrostatic interactions between the gas molecules and graphene surfaces. The charge migration and the other interactions (e.g. Van der Waals interactions and bond/angle interactions) brought from the anionic groups would have a negligible effect on the molecular permeation. Considering the 
dynamic process of molecular permeation through the graphene nanopore, the accumulation of gas molecules on the charge-loaded graphene surfaces is not expected to affect the surface free energy ${ }^{5}$, which determines the ability of adsorbing gas molecules and ultimately affect the efficiency of molecular sieving.

\subsection{MD simulation method}

The MD simulations exploring the selectivity of graphene nanopores are performed in a non-equilibrium system, where the gas mixture $\left(\mathrm{CO}_{2}\right.$ and $\mathrm{N}_{2}$ molecules) is originally arranged in one side of the graphene sheet (feed side). The graphene sheet is located at the center of simulation box with a height of $140 \mathrm{~nm}$. Initially, $500 \mathrm{CO}_{2}$ molecules and $500 \mathrm{~N}_{2}$ molecules are uniformly placed in the feed side. To simulate the porous graphene with a certain pore density, in the directions parallel to the graphene sheet the periodic boundary conditions are applied. The graphene sheet is partially fixed with the flexible pore-rim carbon and hydrogen atoms during the whole simulation period such that the displacement of graphene sheet caused by the molecular collisions is prevented. The partially fixed graphene sheet is slightly different from that in the real systems, where all the graphene atoms have a weak fluctuation caused by the molecular collisions. The temperature of simulation system $(350 \mathrm{~K})$ is equilibrated in the NVT ensemble using the Nosé-Hoover thermostat.

For the all-atom classical MD simulations, the atomic interacting potentials should be considered carefully. For the $\mathrm{C}$ and $\mathrm{H}$ atoms in the graphene sheet, the interactions are modeled by the AIREBO potential ${ }^{6}$; for the $\mathrm{CO}_{2}$ and $\mathrm{N}_{2}$ molecules, they are modeled by the Lennard-Jones potential coupling with a Coulombic term to consider both the Van der Waals and short-rang electrostatic interactions. The Particle-Particle Particle-Mesh method is applied to consider the long-rang electrostatic interactions. The bond and angle interactions in the $\mathrm{CO}_{2}$ and $\mathrm{N}_{2}$ molecules are modeled by the Harmonic potential. For more information of these potential models, one can refer to the Section 2 of Supporting

Information. 


\subsection{Calculation of permeance}

The molecular permeance is obtained based on the time-varying number of molecules in permeate side. The time-varying curves of permeated molecular number $N_{\text {net }}$ can be fitted in the following form, which is obtained based on the relationship between permeation flux and molecular permeance ${ }^{7}$.

$$
N_{\text {net }}=\left(250-N_{\text {al }} / 2\right) \times\left(1-e^{-8.24 \times 10^{10} P \tau}\right)
$$

where $P$ is the molecular permeance $\left(\mathrm{mol} / \mathrm{sm}^{2} \mathrm{~Pa}\right), N_{\mathrm{al}}$ is the average number of adsorbed molecules on both sides of graphene. Therefore, the molecular permeance $P$ can be obtained from the fitted exponent in the time decay of this equation. Then, the selectivity $S$ of $\mathrm{CO}_{2}$ molecules over $\mathrm{N}_{2}$ molecules can be easily obtained as $S=P_{\mathrm{CO} 2} / P_{\mathrm{N} 2}$.

\section{Simulation model}

\subsection{Simulation system}

We perform $\mathrm{MD}$ simulations for the separation of $\mathrm{CO}_{2} / \mathrm{N}_{2}$ mixtures in a nonequilibrium system. In the system, there are 1000 mixed gas molecules, namely $500 \mathrm{CO}_{2}$ molecules and $500 \mathrm{~N}_{2}$ molecules. Initially, the molecules are arranged alternatively and uniformly in the feed side of graphene, while the permeate side is kept in a vacuum, as shown in Figure S1. Graphene is located at the center of the simulation box with a height of $140 \mathrm{~nm}$. Periodic boundary conditions are applied in the $x$ - and $y$-directions (parallel to the graphene surface), while the reflective wall condition is applied in the $z$-direction (perpendicular to the graphene surface). On account of the periodic boundary conditions, we use a graphene sheet with an area of $4 \times 4 \mathrm{~nm}^{2}$ and place the nanopore in the center of graphene to reach a given porosity. The simulation is run in a NVT ensemble with a temperature of $350 \mathrm{~K}$. A simulation period of $1.0 \times 10^{8}$ timesteps (time step is $0.3 \mathrm{fs}$ ) is chosen such that enough gas molecules can transport through the pore but the number of permeated molecules is increased with a relatively fast rate. In such way, a high accuracy in the calculation of molecular permeance can be assured. Based on the ideal gas 
equation ( $p V=N k_{\mathrm{B}} T$ ), the initial pressure of each gas species in the feed side is estimated as 21.1 bar.

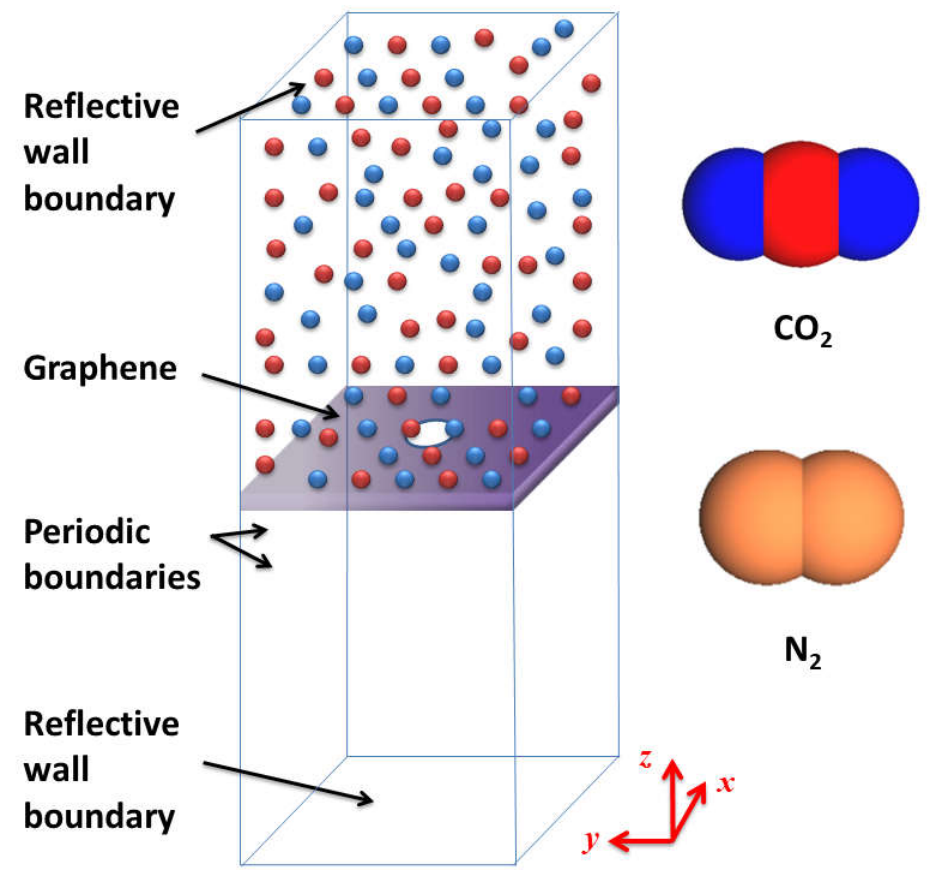

Figure S1 Simulation system adopted in this study, including the non-equilibrium MD simulation box and the atomic models of $\mathrm{CO}_{2}$ and $\mathrm{N}_{2}$ molecules.

\subsection{Atomic interactions}

To model the atomic interactions for $\mathrm{CO}_{2}$ and $\mathrm{N}_{2}$ molecules, a potential model coupling the Lennard-Jones ( $\mathrm{LJ}$ ) interactions and the Coulombic interactions is adopted, as follows:

$\phi\left(r_{i j}\right)=4 \varepsilon\left[\left(\frac{\sigma}{r_{i j}}\right)^{12}-\left(\frac{\sigma}{r_{i j}}\right)^{6}\right]+\frac{C q_{i} q_{j}}{\chi r_{i j}}$

where $\sigma$ is the length scale, $\varepsilon$ is the energy scale, $q_{\mathrm{i}}$ and $q_{\mathrm{j}}$ are the charges on atom $\mathrm{i}$ and atom $\mathrm{j}, C$ is an energy-conversion constant, and $\chi$ is the dielectric constant. Here, the relative dielectric constants for all the atoms are adopted as 1 . The cutoff radius for the short-range atomic interactions is set as $10 \AA$. 
Interaction parameters between cross atoms are obtained by using the LorentzBerthelot mixing rule ( $l$ and $s$ represent two different types of atoms):

$\sigma_{s l}=\frac{\sigma_{s s}+\sigma_{l l}}{2}, \varepsilon_{s l}=\sqrt{\varepsilon_{s s} \varepsilon_{l l}}$

The parameters in Eq. (S2) for the $\mathrm{CO}_{2}$ and $\mathrm{N}_{2}$ molecules are listed in Table S1; the $\mathrm{LJ}$ parameters among the $\mathrm{C}$ atoms in graphene, the functionalized $\mathrm{H}$ atoms and the atoms in the gas molecules are listed in Table S2. The parameters for the cross atoms are estimated by using the Lorentz-Berthelot mixing rule based on the following parameters for C-C interactions: $\sigma=3.40 \AA, \varepsilon=2.413 \times 10^{-3} \mathrm{eV},{ }^{8} \mathrm{H}-\mathrm{H}$ interactions: $\sigma=2.50 \AA, \varepsilon=$ $1.300 \times 10^{-3} \mathrm{eV},{ }^{9}$ and the parameters listed in Table S1. The partial charges on the pore $\operatorname{rim} \mathrm{C}$ and $\mathrm{H}$ atoms are calculated based on the density functional theory by using the DMol3 module in the Material Studio software.

Table S1 Parameters in the potential model Eq. (S2) of gas molecules.

\begin{tabular}{|c|c|c|c|}
\hline & $\varepsilon(\mathrm{eV})$ & $\overline{\sigma(\AA)}$ & charge (e) \\
\hline \multicolumn{4}{|c|}{$\mathrm{CO}_{2}{ }^{10}$} \\
\hline $\mathrm{C}-\mathrm{C}$ & $2.424 \times 10^{-3}$ & 2.757 & 0.6512 \\
\hline $\mathrm{C}-\mathrm{O}$ & $4.101 \times 10^{-3}$ & 2.895 & / \\
\hline $\mathrm{O}-\mathrm{O}$ & $6.938 \times 10^{-3}$ & 3.033 & -0.3256 \\
\hline \multicolumn{4}{|c|}{$\mathrm{N}_{2}{ }^{11}$} \\
\hline $\mathrm{N}-\mathrm{N}$ & $3.126 \times 10^{-3}$ & 3.297 & 0 \\
\hline
\end{tabular}

Table S2 LJ potential parameters for the cross atoms used in the simulations.

\begin{tabular}{ccc}
\hline & $\varepsilon(\mathrm{eV})$ & $\sigma(\AA)$ \\
\hline $\mathrm{C}-\mathrm{N}_{\mathrm{N} 2}$ & $2.746 \times 10^{-3}$ & 3.349 \\
$\mathrm{C}-\mathrm{C}_{\mathrm{CO} 2}$ & $2.418 \times 10^{-3}$ & 3.079 \\
$\mathrm{C}-\mathrm{O}$ & $4.092 \times 10^{-3}$ & 3.217 \\
$\mathrm{H}-\mathrm{N}_{\mathrm{N} 2}$ & $2.016 \times 10^{-3}$ & 2.899 \\
$\mathrm{H}-\mathrm{C} \mathrm{CO} 2$ & $1.775 \times 10^{-3}$ & 2.629 \\
$\mathrm{H}-\mathrm{O}$ & $3.003 \times 10^{-3}$ & 2.767
\end{tabular}




\subsection{Harmonic potential}

The bond stretch and bond angle deformation in the flexible gas molecules $\left(\mathrm{CO}_{2}\right.$ and $\mathrm{N}_{2}$ ) are properly modeled by the harmonic potential. The harmonic bond stretch potential is given by:

$E_{\text {bond }}=K_{\text {bond }}\left(r-r_{0}\right)^{2}$

where $K_{\text {bond }}$ is the bond coefficient and $r_{0}$ is the equilibrium bond distance (bond length).

The harmonic angle deformation potential is given by:

$E_{\text {angle }}=K_{\text {angle }}\left(\theta-\theta_{0}\right)^{2}$

where $K_{\text {angle }}$ is the angle coefficient and $\theta_{0}$ is the equilibrium value of bond angle. The parameters for the bond and angle harmonic potentials can be found in our early work ${ }^{12}$.

\section{Snapshot of molecular permeation}

Here, we give the snapshots of the system at the end of simulation period to have an intuitive impression on the alteration of non-selective graphene nanopore to selective graphene nanopore. Figure $\mathbf{S 2}$ clearly shows that the proportion of $\mathrm{CO}_{2}$ and $\mathrm{N}_{2}$ molecules in the permeate side is obviously different at different surface charge densities. With increasing the surface charge density, the number of $\mathrm{CO}_{2}$ molecules in the permeate side obviously increases while that of $\mathrm{N}_{2}$ molecules decreases, meaning that the selectivity of $\mathrm{CO}_{2}$ molecules over $\mathrm{N}_{2}$ molecules increases gradually. 


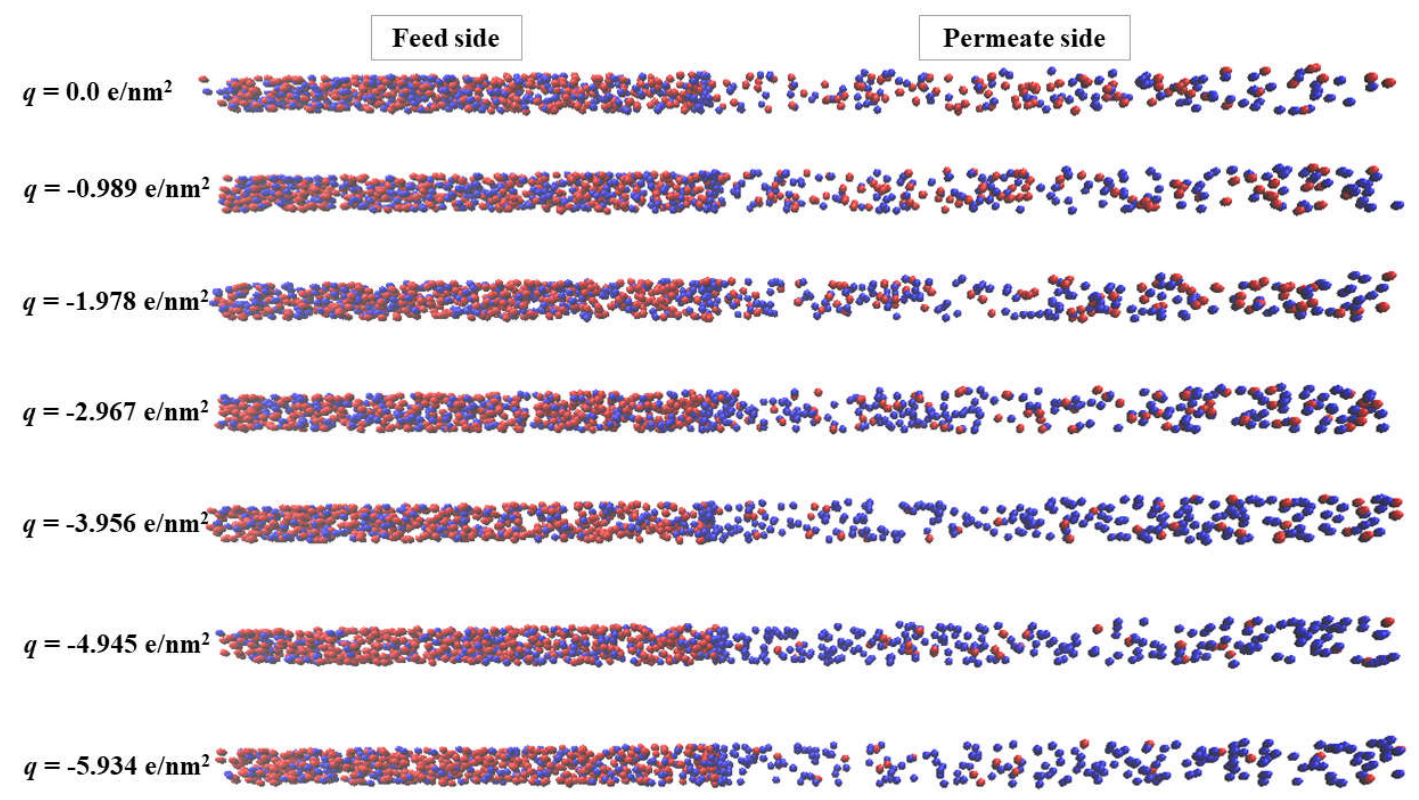

Figure S2 Snapshots of the system at the end of simulation period for different surface charge densities. Blue spheres represent $\mathrm{CO}_{2}$ molecules while red spheres represent $\mathrm{N}_{2}$ molecules, and the graphene atoms are not displayed.

\section{Number of permeated molecules}

Here, Figure S3 displays the time-varying number of permeated molecules for the $\mathrm{CO}_{2}$ and $\mathrm{N}_{2}$ molecules at different surface charge densities. This figure also clearly demonstrates the increase in the selectivity of graphene nanopore with increasing the surface charge density. 

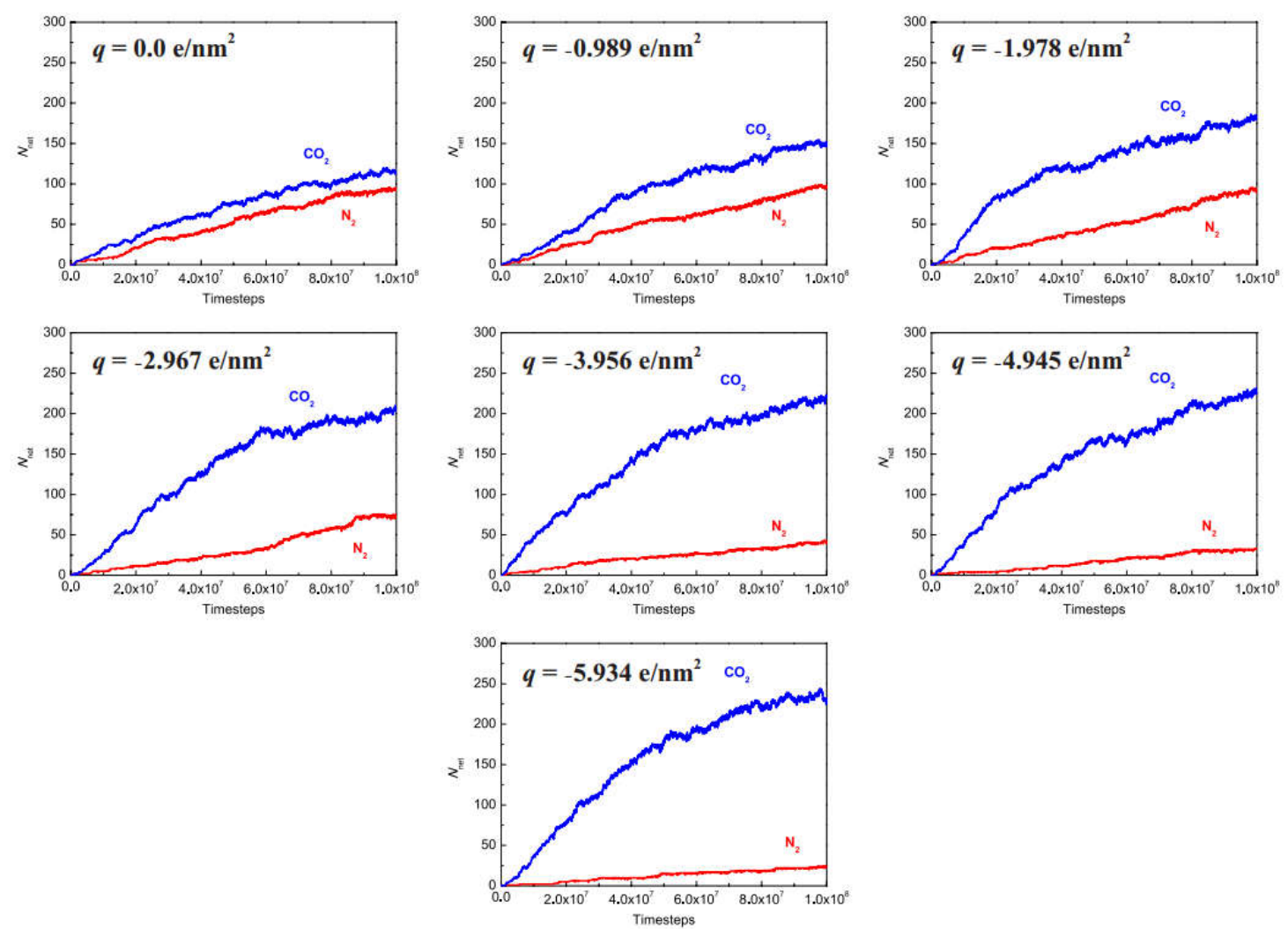

Figure S3 Time-varying number of permeated molecules for the $\mathrm{CO}_{2}$ and $\mathrm{N}_{2}$ molecules at different surface charge densities.

The largest selectivity 22.78 for separating $\mathrm{CO}_{2} / \mathrm{N}_{2}$ gas mixtures is comparable with those identified for the originally selective graphene nanopores by other researchers. Table S3 gives the selectivity of $\mathrm{CO}_{2}$ molecules over $\mathrm{N}_{2}$ molecules for different selective graphene nanopores in other studies.

Table S3 Selectivity of $\mathrm{CO}_{2}$ molecules over $\mathrm{N}_{2}$ molecules for different selective graphene nanopores.

\begin{tabular}{ccccc}
\hline Selectivity & 60 & 11 & $\approx 10$ & 6 \\
\hline Reference & 13 & 14 & 15 & 16 \\
\hline
\end{tabular}




\section{Molecular number density distribution on the graphene surface}

Figure S4 displays the number density distributions of the adsorbed molecules on the graphene surface for the $\mathrm{CO}_{2}$ molecules at different surface charge densities. It can be clearly seen that the low pit zones around the pore disappear as the surface charge density increases.
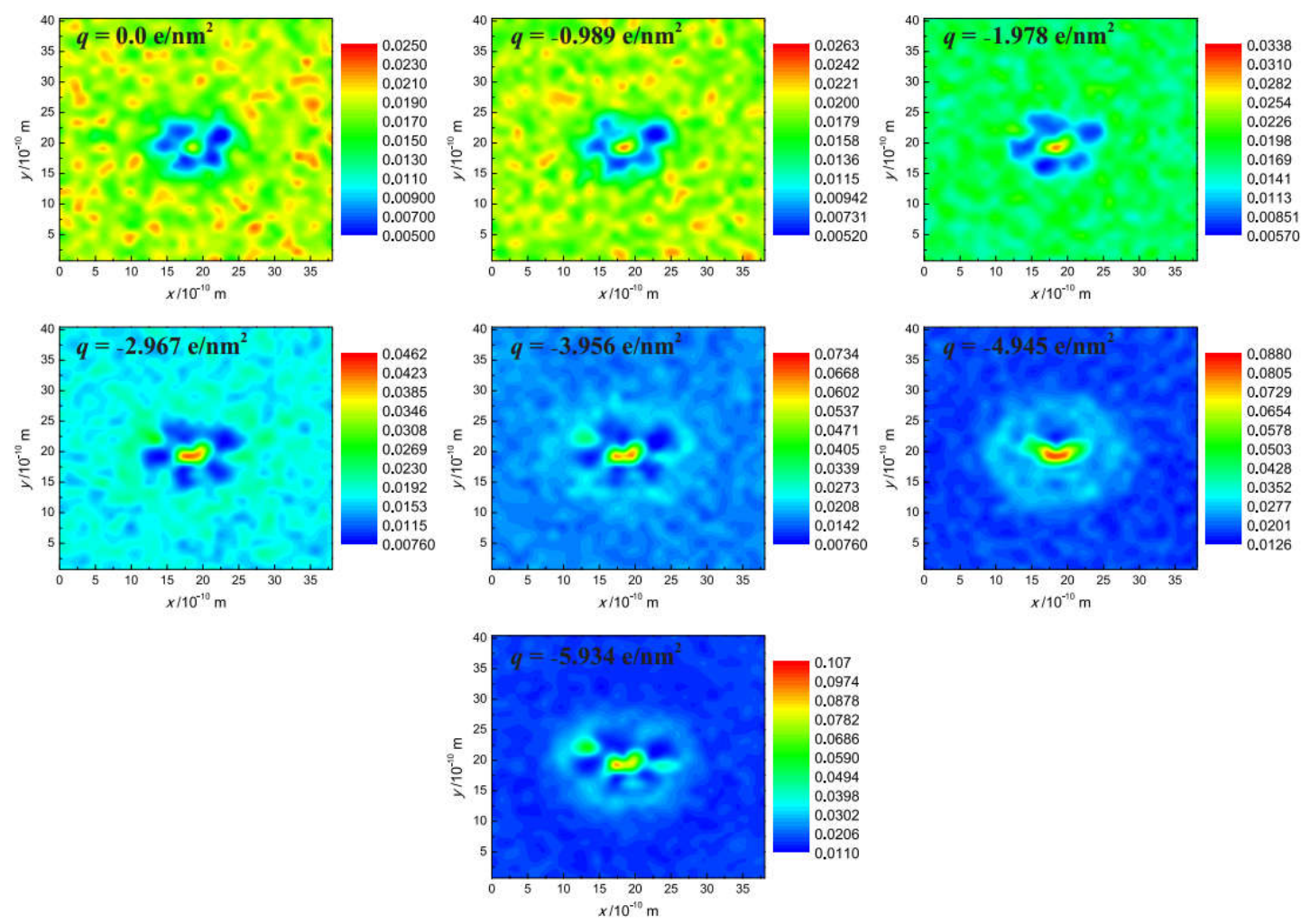

Figure S4 Number density distributions of the adsorbed molecules on the graphene surface for the $\mathrm{CO}_{2}$ molecules. The values shown in these figures are the number of adsorbed molecules on the unit surface with an area of $1.74 \AA^{2}$. 


\section{Reference}

(1) O’Hern, S. C.; Boutilier, M. S. H.; Idrobo, J.-C.; Song, Y.; Kong, J.; Laoui, T.; Atieh, M.; Karnik, R. Selective Ionic Transport through Tunable Subnanometer Pores in Single-Layer Graphene Membranes. Nano Lett. 2014, 14, 1234-1241.

(2) Boutilier, M. S. H.; Jang, D.; Idrobo, J.-C.; Kidambi, P. R.; Hadjiconstantinou, N. G.; Karnik, R. Molecular Sieving across Centimeter-Scale Single-Layer Nanoporous Graphene Membranes. Acs Nano 2017, 11, 5726-5736.

(3) Jang, D.; Idrobo, J.-C.; Laoui, T.; Karnik, R. Water and Solute Transport Governed by Tunable Pore Size Distributions in Nanoporous Graphene Membranes. Acs Nano 2017, 11, 10042-10052.

(4) Kidambi, P. R.; Jang, D.; Idrobo, J.-C.; Boutilier, M. S. H.; Wang, L.; Kong, J.; Karnik, R. Nanoporous Atomically Thin Graphene Membranes for Desalting and Dialysis Applications. Adv. Mater. 2017, 29, 1700277.

(5) Cao, Z.; Tsai, S. N.; Zuo, Y. Y. An Optical Method for Quantitatively Determining the Surface Free Energy of Micro- and Nanoparticles. Anal. Chem. 2019, 91, 12819-12826.

(6) Stuart, S. J.; Tutein, A. B.; Harrison, J. A. A Reactive Potential for Hydrocarbons with Intermolecular Interactions. J. Chem. Phys. 2000, 112, 6472-6486.

(7) Sun, C.; Bai, B. Molecular Sieving through a Graphene Nanopore: NonEquilibrium Molecular Dynamics Simulation. Sci. Bull. 2017, 62, 554-562.

(8) Du, H. L.; Li, J. Y.; Zhang, J.; Su, G.; Li, X. Y.; Zhao, Y. L. Separation of Hydrogen and Nitrogen Gases with Porous Graphene Membrane. J. Phys. Chem. C 2011, $115,23261-23266$.

(9) Liu, H.; Chen, Z.; Dai, S.; Jiang, D.-e. Selectivity Trend of Gas Separation through Nanoporous Graphene. J. Solid State Chem. 2015, 224, 2-6.

(10) Liu, H.; Dai, S.; Jiang, D. Insights into $\mathrm{CO}_{2} / \mathrm{N}_{2}$ Separation through Nanoporous Graphene from Molecular Dynamics. Nanoscale 2013, 5, 9984-9987.

(11) Chae, K.; Violi, A. Mutual Diffusion Coefficients of Heptane Isomers in Nitrogen: A Molecular Dynamics Study. J. Chem. Phys. 2011, 134, 044537. 
(12) Sun, C.; Wen, B.; Bai, B. Application of Nanoporous Graphene Membranes in Natural Gas Processing: Molecular Simulations of $\mathrm{CH}_{4} / \mathrm{CO}_{2}, \mathrm{CH}_{4} / \mathrm{H}_{2} \mathrm{~S}$ and $\mathrm{CH}_{4} / \mathrm{N}_{2}$ Separation. Chem. Eng. Sci. 2015, 138, 616-621.

(13) Schrier, J. Carbon Dioxide Separation with a Two-Dimensional Polymer Membrane. Acs Appl. Mater. Interf. 2012, 4, 3745-3752.

(14) Shan, M.; Xue, Q.; Jing, N.; Ling, C.; Zhang, T.; Yan, Z.; Zheng, J. Influence of Chemical Functionalization on the $\mathrm{CO}_{2} / \mathrm{N}_{2}$ Separation Performance of Porous Graphene Membranes. Nanoscale 2012, 4, 5477-5482.

(15) Wu, T.; Xue, Q.; Ling, C.; Shan, M.; Liu, Z.; Tao, Y.; Li, X. Fluorine-Modified Porous Graphene as Membrane for $\mathrm{CO}_{2} / \mathrm{N}_{2}$ Separation: Molecular Dynamic and FirstPrinciples Simulations. J. Phys. Chem. C 2014, 118, 7369-7376.

(16) Wang, Y.; Yang, Q.; Zhong, C.; Li, J. Theoretical Investigation of Gas Separation in Functionalized Nanoporous Graphene Membranes. Appl. Surf. Sci. 2017, 407, 532-539. 